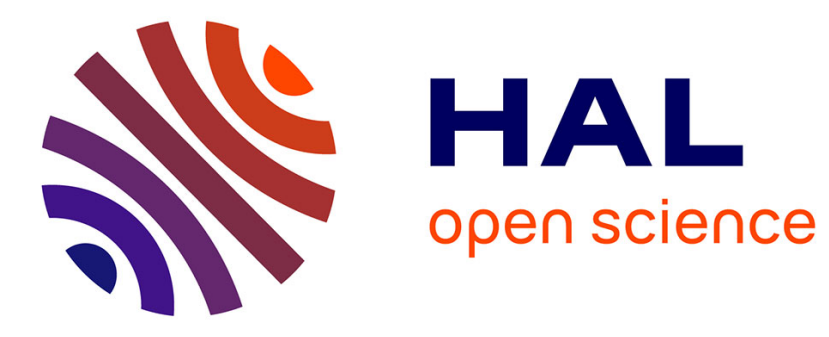

\title{
Mutual Insurance Networks in Communities
}

Pascal Billand, Christophe Bravard, Sudipta Sarangi

\section{To cite this version:}

Pascal Billand, Christophe Bravard, Sudipta Sarangi. Mutual Insurance Networks in Communities.

2012. halshs-00768430

\section{HAL Id: halshs-00768430 \\ https://shs.hal.science/halshs-00768430}

Preprint submitted on 21 Dec 2012

HAL is a multi-disciplinary open access archive for the deposit and dissemination of scientific research documents, whether they are published or not. The documents may come from teaching and research institutions in France or abroad, or from public or private research centers.
L'archive ouverte pluridisciplinaire HAL, est destinée au dépôt et à la diffusion de documents scientifiques de niveau recherche, publiés ou non, émanant des établissements d'enseignement et de recherche français ou étrangers, des laboratoires publics ou privés. 


\section{Mutual Insurance Networks in Communities}

Pascal Billand, Christophe Bravard, Sudipta Sarangi

December 2012 


\section{GATE Groupe d'Analyse et de Théorie Économique Lyon-St Étienne}

93, chemin des Mouilles 69130 Ecully - France

Tel. +33(0)4 72866060

Fax $+33(0) 472866090$

6, rue Basse des Rives 42023 Saint-Etienne cedex 02 - France

Tel. +33 (0)4 77421960

Fax. +33 (0)4 77421950

Messagerie électronique / Email : gate@gate.cnrs.fr

Téléchargement / Download : http://www.gate.cnrs.fr - Publications / Working Papers 


\title{
Mutual Insurance Networks in Communities*
}

\author{
Pascal Billand $^{a},{\text { Christophe } \text { Bravard }^{b}, \text { Sudipta Sarangi }}^{c}$
}

December 19, 2012

${ }^{a}$ Université de Lyon, Lyon, F-69007, France ; CNRS, GATE Lyon Saint-Etienne, Ecully, F-69130, France ; Universit Jean Monnet, Saint-Etienne, F-42000, France.

email: pascal.billand@univ-st-etienne.fr.

${ }^{b}$ Université Grenoble 2 ; UMR 1215 GAEL, F-38000 Grenoble, France ; CNRS, GATE Lyon St

Etienne, Saint-Etienne, F-42000, France.email: christophe.bravard@univ-st-etienne.fr.

${ }^{c} D I W$ Berlin and Louisiana State University. email: sarangi@lsu.edu

\begin{abstract}
We study the formation of mutual insurance networks in a model where every agent who obtains more resources gives a fixed amount of resources to all agents who have obtained less resources. The low resource agent must be directly linked to the high resource agent to receive this transfer. We identify the pairwise stable networks and efficient networks. Then, we extend our model to situations where agents differ in their generosity with regard to the transfer scheme. We show that there exist conditions under which in a pairwise stable network agents who provide the same level of transfers are linked together, while there are no links between agents who provide high transfers and agents who provide low transfers.
\end{abstract}

Keywords: Mutual insurance networks, Pairwise stable networks, Efficient networks. JEL classification: C72, D81, D8.

*This paper has benefitted from the comments of Matt Jackson, Francis Bloch and participants at the Networks and Development Conference in Louisiana State University. 


\section{Introduction}

A growing body of evidence has shown that, while household income in developing countries varies greatly, consumption is remarkably smooth (e.g., Townsend, 1994, Paxson, 1992, Jacoby and Skouas, 1997). Given the absence of formal insurance especially in the rural areas, this suggests that informal institutions allow households to counter the effects of income variation. In this paper we study the formation of informal mutual insurance networks building on several stylized facts about them. Our first key feature will be to incorporate the notion that mutual insurance relationships are not a village level phenomenon. Indeed, as has been well documented in the literature on social networks, in times of need individuals do not rely on the entire village, instead they seek help primarily from friends and family (see Fafchamps and Lund, 2003, and Wellman and Currington, 1988). Hence in our model mutual insurance occurs among individuals who are directly connected to each other. Consequently, one may ask what the structure of stable networks will be at the community level. How exactly will they differ from the socially optimal networks? Finally, following the empirical literature we also want to allow for the fact that the sharing of resources in times of need is not equal (Townsend, 1994). In fact to the best of our knowledge, the formation of mutual insurance networks to answer this question has not been addressed before. So our goal is to determine the structure of stable and efficient networks where not all agents obtain the same resources.

In line with these empirical observations, we build a benchmark model where mutual insurance takes place between pairs of risk averse individuals in a village or a small community. The network of relationships between individuals constitutes the mutual insurance network that allows agents to obtain insurance against resources fluctuations. A specific feature of our setting is the way agents "share" their resources (and hence the risk): individuals who draw high resources give a fixed amount of resources to individuals who draw low resources in their immediate neighborhood in the network. Thus, in our setting, agents do not engage in equal sharing of resources. This type of sharing mechanism has two desirable features: (i) it ensures that individuals who draw high resources can still transfer resources to individuals who draw low resources, and (ii) individuals who draw high resources always obtain higher benefits than individuals who draw low resources. 
In our benchmark model, we assume that each agent obtains random resources which take on two values: high or low. If a person draws the high endowment state, then she gives an amount $\delta>0$ of resources to each of her neighbors (agents with whom she has established a bilateral risk-sharing agreement) that has drawn the low endowment state. Conversely, if a person draws the low endowment state, then she obtains an amount $\delta$ of resources from each of her neighbors who has drawn the high endowment state. Note that such a mutual insurance network exposes agents to the risk of their neighbors. Indeed, if two individuals decide to insure each other, then each of them increases her chances of obtaining a satisfying payoff when her own resources are low, but also increases her chances of reducing her payoffs when her own resources are high.

In our model, establishing bilateral mutual insurance agreements (links) is costly, i.e., the cost of an agreement (a link) between two individuals depends on the number of agreements established by them. So, the cost incurred by agent $i$ when she forms a link with agent $j$ increases when $i$ and $j$ have formed more links. More precisely, we assume that the marginal cost of individual $i$, when she forms a link with an individual $j$, is increasing with the number of links she has formed and the number of links that agent $j$ has formed. This captures the idea that a mutual insurance agreement between two agents requires the agents to spend a minimal amount of time on it. The more links they have already formed, the less time they have to spend on any additional link, and so the higher is the cost of time. It follows that the cost of an additional link increases with the number of links. Similarly, for the benefits of a link, we assume that each additional link is less valuable than the previous one.

Using this framework, we examine the formation of mutual insurance links and ask what structures will emerge when agents cannot coordinate link formation across the entire population. We use pairwise stability as equilibrium concept (see Jackson and Wolinsky, 1996). In a pairwise stable network no pair of unlinked agents has an incentive to reach a mutual insurance agreement (add a link) and no individual has an incentive to break a mutual insurance agreement (remove one of her links). We contrast pairwise stable networks with the efficient networks for mutual insurance agreements. Efficient network is one which maximizes the amount of total payoff obtained by agents. 
In the basic model, we have several findings.

- First, we establish that there exist pairwise stable networks, in which individuals are in asymmetric situations relative to the risk they support. Moreover, in these pairwise stable networks agents who obtain the smallest amount of insurance are always linked together while agents who have the highest amount of insurance do not form links with the low insurance agents.

- Second, in efficient networks agents always obtain similar amounts of insurance. Consequently, we observe the possibility of a conflict between pairwise stable networks and efficient networks. We conclude that the mutual insurance mechanism described here does not always lead to efficient networks.

Finally, we extend the basic model to situations where agents do not have the same preferences with respect to the generosity of the transfer scheme. More precisely, we assume that there exist two types of agents: the generous ones who are more giving than the miserly ones. We establish that there exist conditions under which, in a pairwise stable network, generous agents are linked together, miserly agents are linked together, but there are no links between these two types of agents. This kind of pairwise stable networks are compatible with a result stressed by several empirical studies: the majority of transfers takes place only between sub-groups of agents (see Rosenzweig, 1988, and Udry, 1994).

A recent theoretical literature about revenue sharing in developing economies examines the formation of risk-sharing networks. Bramoulle and Kranton (2006 and 2007) deal with the formation of risk-sharing networks and discuss the stability/efficiency dilemma of risksharing networks. A distinctive aspect of their work is that after the income realization occurs, linked pairs of agents meet (sequentially and randomly) and share their current money holding equally. The authors show that with many rounds of such meetings, an individuals money holding converges to the mean of realized income in her group, ${ }^{1}$ that is in a group there is equal revenue sharing among individuals. Belladj and Deroian [?] also examine a situation where the bilateral partial risk-sharing rule is such that neighbors share equally a part of their

\footnotetext{
${ }^{1}$ In their paper, a group consists of agents who are both directly or indirectly linked.
} 
revenue. However, they focus on the impact of informal risk-sharing on risk taking incentives when transfers are organized through a social network. By contrast, in our paper we deal with situations where individuals do not engage in equal income sharing. In particular, after income sharing, an individual who has initially obtained high income always ends up better than an individual who has obtained low income. There is an interesting difference between our paper and the Bramoulle and Kranton papers concerning externality of the links. In our paper, when an agent $i$ forms a link with an agent $j$, this link has a negative impact on the utility of $i$ 's neighbors (there is a negative externality), since agent $i$ will have less time to spend on the relation with her neighbors (idem for $j$ 's neighbors). It follows that it can be that two agents have an incentive to form a link, and this link decreases the social welfare. In the Bramoulle and Kranton model, when an agent $i$ forms a link with an agent $j$, this link has a positive impact on the expected utility of $i$ 's neighbors. Indeed, in this model there is equal income sharing between all the agents of the group. Therefore due to the additional link between $i$ and $j, i$ 's neighbors will share their income with additional agents ( $j$ and her neighbors) and their expected payoff will increase. Therefore, it can be that two agents have no incentive to form a link, and this link increases the social welfare.

Some papers explain partial risk-sharing by self-enforcing mechanisms on networks (Bloch et al., 2008). ${ }^{2}$ These models consider that individuals can use their links to punish individuals who deviate from the insurance scheme. For instance, if an agent deviates from the insurance scheme (ie. fails to transfer money to directly connected agents that have negative income shocks), the victim will communicate such behavior to connected agents who in turn will terminate the insurance scheme with the deviating household as a punishment. In this paper, we do not deal with the self-enforcement mechanism problem. Indeed, we assume that establishing a relation is costly and the relation commits the parties to future resources sharing, say, due to a social norm or a social punishment in case of non-sharing ${ }^{3}$. More precisely, we assume that the self-enforcement mechanism problem is solved when agents invest enough time and

${ }^{2}$ This literature extends the literature about the robustness of mutual insurance (see for instance Genicot and Ray, 2003).

${ }^{3}$ This kind of relation can be illustrated with the marriage of daughters in India which are arranged to maximize gains from risk sharing, see Rosenzweig and Stark, 1989. 
resources in the informal insurance agreements. ${ }^{4}$ Our assumptions about the cost of a bilateral insurance agreement (a link), take into account the fact that agents have a limited amount of time.

The rest of the paper is organized as follows. In section 2, we present the definitions and the basic model setup. In section 3, we examine pairwise stable networks and efficient networks in the basic model context. In this section, we deal with specific benefits functions, but our results concerning the pairwise stable networks and the efficient networks are always true when the expected benefits function of each agent $i$ is concave, and if the marginal cost incurred by $i$, when she forms a link with $j$, is increasing with the number of links formed by $i$ and with the number of links formed by by $j$. In section 4 , we extend the basic model to situations where agents do not have the same preferences with respect to the generosity of the transfer scheme. In section 5 , we conclude.

\section{Basic model setup}

Let $N=\{1, \ldots, n\}$ be a community of $n, n \geq 3$, ex ante identical agents. Agents receive an endowment and consume resources. Each agent's endowment is a random variable that takes on two values. The low endowment state is called state 0 while the high endowment state is called state 1 . Each agent $i$ obtains an endowment 0 in state 0 while she obtains $\Theta>0$ in state 1 . This state occurs with probability $p>0$ while the low endowment state occurs with probability $1-p>0$. The realizations of endowment are independent and identical across the agents.

\section{$2.1 \quad$ Networks}

To model bilateral mutual insurance agreements in a small population, we use tools from the theory of networks. Although the agreements themselves are bilateral, the amount of resources consumed by each agent depends on how many other agents she is connected to,

\footnotetext{
${ }^{4}$ The time an agent invests in the relation and the social punishment in case of non-sharing are related since a bilateral relation in which agents have invested a lot of time can be more easily observed by the peers.
} 
and the endowments of these agents. Hence tools from network theory are useful for modeling such bilateral insurance networks. In the model, we assume that individuals $i$ and $j$ can make a mutual insurance agreement by forming a costly link between themselves. This assumption reflects the idea that there are always some costs (time at the least) to build a relationship.

We represent links and a network of links with the following notation: A network $g$ is a $n \times n$ matrix, where $g_{i j}=1$ when $i$ and $j$ have formed a link (i.e., have established a risksharing agreement) and $g_{i j}=0$ otherwise. We assume that risk-sharing relations are mutual, so that $g_{i j}=g_{j i}$. By convention, $g_{i i}=0$. Let $g+g_{i j}$ denote the network obtained by replacing $g_{i j}=0$ in $g$ by $g_{i j}=1$. Similarly, let $g-g_{i j}$ denote the network obtained by replacing $g_{i j}=1$ in $g$ by $g_{i j}=0$. We say that there is a chain between two agents $i$ and $j$ in the network $g$ if there exists a sequence of agents $i_{1}, \ldots, i_{k}$ such that $g_{i i_{1}}=g_{i_{1} i_{2}}=g_{i_{2} i_{3}}=\ldots=g_{i_{k} j}=1$. A subset of agents is connected if there is a chain between any two agents in the subset. A component of the network $g$ is a maximal connected subset of agents.

The empty network is the network where all agents have formed no links. The complete network is the network where each agent has formed links with all other agents. The star is a network where an agent, say $i$, has formed a link with all others agents while the other agents have not formed any link except with $i$. A $k$-regular network is a network where all agents have formed exactly $k$ links. An almost-k-regular network is a network where all agents but one have formed $k$ links. The agent who is the exception has formed either $k+1$ links, or $k-1$ links. We illustrate the notions of star, k-regular network, and almost-k-regular network in Figure 1. More precisely, in Figure 1.b. we have drawn a 2-regular network, that is a network where all agents are involved in 2 links; in Figure 1.c., we have drawn an almost-3-regular network where all agents are involved in 3 links but agent 1 has formed 4 links.

In the following, the neighbors of agent $i$, that is agents with whom $i$ has formed a link, will play a crucial role. Hence we define $N_{i}(g)=\left\{j \in N \mid g_{i j}=1\right\}$ as the set of the neighbors of $i$. We let $n_{i}(g)=\left|N_{i}(g)\right|$. 


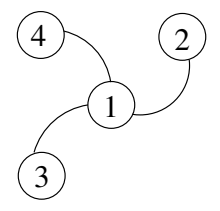

a. Star

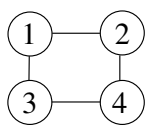

b. 2-regular network

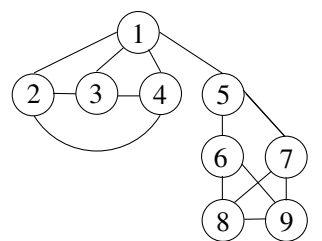

c. almost-3-regular network

Figure 1: Networks architectures

\subsection{Mutual insurance agreement networks: Payoffs}

Having described the set of players and their strategies, we now ask: Given a network $g$, how are expected payoffs determined under different endowment realizations in the network? We consider a benchmark model where each agent $i$ commits to help the partners with whom she is linked when she obtains high resources, while her partners help her when she obtains low resources. More precisely, if agent $i$ draws state 1 , then she gives $\delta \in(0,1)$ to each of her neighbors who have drawn state $0 .{ }^{5}$ Conversely, if she draws state 0 , then each of her neighbors who has drawn state 1 gives her $\delta$. This assumption underscores the fact that $e x$ ante every agent is identical and Wellman and Currington, 1988, gets the same resources in the high and low states. It follows that in our model, agents may receive different amounts of transfers depending on the network architecture.

In this paper we assume that the payoff obtained by an agent, say $i$, can be divided into two parts.

1. The benefits part which involves uncertainty captures the fact that each additional link formed by $i$ allows her to obtain additional insurance when she draws state 0 , and the fact that $i$ has to insure more agents when she draws state 1 .

2. The costs part which involves no uncertainty captures the fact that links are costly, with additional links being more costly.

\footnotetext{
${ }^{5}$ Here, we assume that the transfer amount $\delta$ comes from some kind of social norm. Since our goal is to study the architectures of the mutual insurance agreements networks, we do not explicitly model where $\delta$ comes from.
} 
We now present these two parts of an agent's payoff function.

Benefits. Let $b^{g}(x, y)$ be the benefits that an agent $i$, with $n_{i}(g)$ neighbors, obtains when she draws $x, x \in\{0,1\}$, and $y$ of her neighbors draws 1 , with $y \in\left\{0, \ldots, n_{i}(g)\right\}$. A reasonable benefits function should satisfy the following properties:

- $(\mathrm{P} 1) b^{g}(0, \cdot)$ and $b^{g}(1, \cdot)$ are increasing;

- $(\mathrm{P} 2) b^{g}(0, \cdot)$ and $b^{g}(1, \cdot)$ are concave; ${ }^{6}$

- (P3) For $n_{i}(g)=n-1, b^{g}(0, n-1)-b^{g}(0, n-2)>b^{g}(1,1)-b^{g}(1,0)$.

Property $\mathrm{P} 1$ states that the benefits of an agent $i$ are increasing as more of her neighbors draw state 1, regardless of what state she draws. Indeed, in her low endowment state she receives more insurance, and in the high endowment state she has to insure fewer people. When agent $i$ draws state $0, \mathrm{P} 2$ just states that the benefits of each additional unit of resources decline with the amount of resources. When agent $i$ draws state 1, P2 means that the marginal loss, $\left|b^{g}(1, k-1)-b^{g}(1, k)\right|$, that agent $i$ incurs when $k-1$ neighbors instead of $k$ draw state 1 is higher than the marginal loss, $\left|b^{g}(1, k)-b^{g}(1, k+1)\right|$, that agent $i$ incurs when $k$ neighbors instead of $k+1$ draws state 1 . Given that the benefits functions are concave, it follows from Property P3 that the maximal marginal benefits associated with $b^{g}(1, \cdot)$ is smaller than the minimal marginal benefits associated with $b^{g}(0, \cdot)$. Indeed, the minimal marginal benefits associated with $b^{g}(0, \cdot)$ is $b^{g}(0, n-1)-b^{g}(0, n-2)$ while the maximal marginal benefits associated with $b^{g}(1, \cdot)$ is $b^{g}(1,1)-b^{g}(1,0)$.

Working with a general payoff function that satisfies these three properties in the context of a network formation problem is hard. Hence from now on we deal with specific benefits functions that satisfy these properties and make the algebra easier. ${ }^{7}$ We now explain how these benefits functions summarize the payoffs of an agent $i$, given her neighborhood $n_{i}(g)$.

\footnotetext{
${ }^{6}$ Since each player gives the same amount $\delta$ when she helps another player, we define the concavity with the number of players, instead of the amount of resources, in order to simplify the notation.

${ }^{7}$ Note that in our setting, all benefits functions which lead to a concave expected payoff function will yield the same qualitative results for pairwise stable networks and efficient networks.
} 
First, the benefits function $b^{g}(0, k)$ which captures the benefits that $i$ obtains in $g$ when she draws state 0 and $k$ agents in her neighborhood draw state 1 is:

$$
b^{g}(0, k)=\sum_{j=0}^{k}\left(\delta a_{0}\right)^{j}
$$

where $\delta a_{0} \in(0,1)$. The benefits function $b^{g}(0, \cdot)$ is increasing since $b^{g}(0, k)-b^{g}(0, k-1)=$ $\left(\delta a_{0}\right)^{k}>0$, for $k \in\{1, \ldots, n-1\}$, that is the marginal benefits associated with help provided by the $k^{\text {th }}$ neighbor is equal to $\left(\delta a_{0}\right)^{k}>0$. This simple geometric form, which captures the transfers received by agent $i$, ensures concavity of $b^{g}(0, \cdot)$ since $\left[b^{g}(0, k+1)-b^{g}(0, k)\right]-$ $\left[b^{g}(0, k)-b^{g}(0, k-1)\right]=\left(\delta a_{0}\right)^{k+1}-\left(\delta a_{0}\right)^{k}=\left(\delta a_{0}-1\right)\left(\delta a_{0}\right)^{k}<0$, for $k \in\{1, \ldots, n-2\}$. To sum up, $b^{g}(0, \cdot)$ satisfies P1 and P2.

Second, the benefits function $b^{g}(1, k)$ which captures the payoff that agent $i$ obtains in $g$ when she draws state 1 and $k$ agents in her neighborhood draw state 1 is:

$$
b^{g}(1, k)=\Theta+\theta-\theta \sum_{j=0}^{n_{i}(g)-k}\left(\delta a_{1}\right)^{j},
$$

where $\delta a_{1}>1$. The benefits function $b^{g}(1, \cdot)$ is increasing since $b^{g}(1, k)-b^{g}(1, k-1)=$ $\theta\left(\delta a_{1}\right)^{n_{i}(g)-k+1}>0$, for $k \in\{1, \ldots, n-1\}$, that is the marginal loss incurred by agent $i$ due to the help provided to the $\left(n_{i}(g)-k+1\right)^{\text {th }}$ neighbor is equal to $\theta\left(\delta a_{1}\right)^{n_{i}(g)-k+1}>0$. Moreover, $b(1, \cdot)$ is concave since $\left[b^{g}(1, k+1)-b^{g}(1, k)\right]-\left[b^{g}(1, k)-b^{g}(1, k-1)\right]=\theta\left(\delta a_{1}\right)^{n_{i}(g)-k}-$ $\theta\left(\delta a_{1}\right)^{n_{i}(g)-k+1}=\theta\left(1-\delta a_{1}\right)\left(\delta a_{1}\right)^{n_{i}(g)-k}<0$, for $k \in\{1, \ldots, n-2\}$. To sum up, $b^{g}(1, \cdot)$ satisfies $\mathrm{P} 1$ and $\mathrm{P} 2$.

Finally, we combine these two benefits functions into a unique benefits function. The benefits that agent $i$ obtains when she draws state $x, x \in\{0,1\}$, and $k$ of her neighbors draw state 1 are summarized by the following function:

$$
b^{g}(x, k)=(\Theta+\theta+1) x-1+\sum_{j=0}^{k}(1-x)\left(\delta a_{0}\right)^{j}-\theta \sum_{j=0}^{n_{i}(g)-k} x\left(\delta a_{1}\right)^{j},
$$

with $\Theta>\theta \sum_{j=1}^{n-1}\left(\delta a_{1}\right)^{j}+\sum_{j=1}^{n-1}\left(\delta a_{0}\right)^{j}$, and $\left(\delta a_{1}\right)^{n}<(1 / \theta)\left(\delta a_{0}\right)^{n}$. The first inequality, $\Theta>$ $\theta \sum_{j=1}^{n-1}\left(\delta a_{1}\right)^{j}+\sum_{j=1}^{n-1}\left(\delta a_{0}\right)^{j}$, implies that agents who draw 1 always obtain higher benefits than agents who draw 0 . Property P3 follows the condition $\left(\delta a_{1}\right)^{n}<(1 / \theta)\left(\delta a_{0}\right)^{n}$, since we 
have $b^{g}(0, n-1)-b^{g}(0, n-2)=\left(\delta a_{0}\right)^{n-1}>\theta\left(\delta a_{1}\right)^{n-1} \geq b^{g}(1,1)-b^{g}(1,0)$. It is worth noting that $b^{g}(0,0)=0$ : if agent $i$ obtains the low value and no neighbors help her, then she obtains zero benefits. ${ }^{8}$ Similarly, $b^{g}\left(1, n_{i}(g)\right)=\Theta$ : if agent $i$ draws 1 and helps no neighbors, then she obtains benefits equal to $\Theta$.

We now define the expected neighborhood benefits (ENB) function, $B_{i}(g)$, which captures the expected benefits obtained by an agent $i$ given her neighborhood $n_{i}(g)$. We have:

$$
\begin{aligned}
B_{i}(g)=\phi\left(n_{i}(g)\right)= & p \sum_{k=0}^{n_{i}(g)}\left(\begin{array}{c}
n_{i}(g) \\
k
\end{array}\right) p^{k}(1-p)^{n_{i}(g)-k} b^{g}(1, k) \\
& +(1-p) \sum_{k=0}^{n_{i}(g)}\left(\begin{array}{c}
n_{i}(g) \\
k
\end{array}\right) p^{k}(1-p)^{n_{i}(g)-k} b^{g}(0, k),
\end{aligned}
$$

where $\left(\begin{array}{l}x \\ y\end{array}\right)$ is just the probability of $y$ high resources out of $x$ draws. $^{9}$

Costs of links. Informal insurance arrangements are potentially limited by the presence of various incentive constraints. As a first cut, it appears that the most important constraint arises from the fact that these arrangements are informal, i.e., not written on legal paper. It follows that they will be honored only if agents involved in such a relationship invest time. Since each agent has a limited amount of time, the costs for agent $i$ of forming an additional link with some agent $j$ should increase with the number of links formed by agent $i$. Moreover, these costs should also increase with the number of links formed by agent $j$. Indeed, it is more difficult to establish a relationship with an agent who already has numerous links since she has less time available. ${ }^{10}$ We capture these ideas through the following cost function for link formation:

$$
C_{i}(g)=f_{1}\left(n_{i}(g)\right)+\sum_{\ell \in N_{i}(g)} f_{2}\left(n_{\ell}(g)\right)
$$

\footnotetext{
${ }^{8}$ In our context, the fact that benefits equal 0 does not mean that an agent obtains no income. We can easily rescale the benefits function so that agents obtain non-null benefits when they draw the low endowment state and do not help any agents.

${ }^{9}$ Equation 2 illustrates the problem of using a general payoff function. It is the presence of the binomial formula in this expression that makes it necessary for us to use a specific payoff function.

${ }^{10}$ Another option that makes such informal arrangements feasible is the threat of punishment as in Bloch, Genicot and Ray, 2008.
} 
where $f_{1}(\cdot)$ and $f_{2}(\cdot)$ are strictly increasing and convex functions. In addition, $f_{1}(0)=0$. We set $\Delta f_{1}\left(n_{i}(g)+1\right)=f_{1}\left(n_{i}(g)+1\right)-f_{1}\left(n_{i}(g)\right)$. Given this cost function, an additional link formed with agent $j$ induces a cost for agent $i$ equal to

$$
C_{i}\left(g+g_{i j}\right)-C_{i}(g)=\Delta f_{1}\left(n_{i}(g)+1\right)+f_{2}\left(n_{j}(g)+1\right)
$$

Since $f_{1}(\cdot)$ is strictly increasing and $f_{2}(\cdot)>0, C_{i}\left(g+g_{i j}\right)-C_{i}(g)>0$.

Expected payoffs function. The expected payoff function, $U_{i}(g)$, of each agent $i$, given the network $g$, is the difference between the ENB function and the cost function of forming links:

$$
U_{i}(g)=B_{i}(g)-C_{i}(g)=\phi\left(n_{i}(g)\right)-\left(f_{1}\left(n_{i}(g)\right)+\sum_{\ell \in N_{i}(g)} f_{2}\left(n_{\ell}(g)\right)\right)
$$

\subsection{Pairwise stable networks and efficient networks}

A network $g$ is pairwise stable if no unlinked agents would benefit from adding a link in $g$ and if no agent would benefit from severing one of her existing links in $g$. Formally, we have (i) for all $g_{i j}=1, U_{i}(g) \geq U_{i}\left(g-g_{i j}\right)$ and $U_{j}(g) \geq U_{j}\left(g-g_{i j}\right)$; and (ii) for all $g_{i j}=0$, if $U_{i}(g)<U_{i}\left(g+g_{i j}\right)$, then $U_{j}(g)>U_{j}\left(g+g_{i j}\right){ }^{11}$

An efficient network is one that maximizes the sum of the expected payoffs of the agents. Let $W(g)=\sum_{i \in N} U_{i}(g)$ be the total expected payoffs obtained in a network $g$. A network $g^{e}$ is efficient if $W\left(g^{e}\right) \geq W(g)$ for all networks $g$.

\section{Pairwise stable networks and efficient networks in the basic model}

First, we analyze the EBN function. Second, we study pairwise stable networks. Third, we turn to efficient networks.

\footnotetext{
${ }^{11}$ This definition is given by Jackson and Wolinsky, 1996.
} 


\subsection{Analysis of the expected neighborhood benefits function}

The next two propositions provide useful properties of the ENB function. We provide a sketch of proof of these propositions in the appendix.

Proposition 1 Suppose that the benefits function is given by equation 1. Then, the expected neighborhood benefits function of agent $i$ is increasing with the number of links she has formed.

Proposition 1 states that if links were costless, then agents would like to have $n-1$ neighbors in order to obtain maximal insurance. This result captures the fact that agents are risk averse.

Proposition 2 Suppose that the benefits function is given by equation 1. Then, the expected neighborhood benefits function of agent $i$ is strictly concave in the number of links she has formed.

This proposition implies that the marginal ENB that an agent $i$ obtains from an additional link decreases with the number of links she has formed. Consequently, if the cost of forming links was constant, then the incentive of an agent to form an additional link would decrease with the number of links she has formed.

Proposition 2 allows us to characterize some properties of the marginal payoffs, $\Delta U_{i}\left(g, g_{i j}\right)=$ $U_{i}\left(g+g_{i j}\right)-U_{i}(g)$, obtained by agent $i$ in a network $g$ when she forms an additional link with agent $j$. We have

$$
\begin{aligned}
\Delta U_{i}\left(g, g_{i j}\right) & =B_{i}\left(g+g_{i j}\right)-C_{i}\left(g+g_{i j}\right)-\left(B_{i}(g)-C_{i}(g)\right) \\
& =\phi\left(n_{i}(g)+1\right)-\phi\left(n_{i}(g)\right)-\left(f_{1}\left(n_{i}(g)+1\right)-f_{1}\left(n_{i}(g)\right)+f_{2}\left(n_{j}(g)+1\right)\right)
\end{aligned}
$$

Let $\Delta \phi\left(n_{i}(g)+1\right)=\phi\left(n_{i}(g)+1\right)-\phi\left(n_{i}(g)\right)$. Using the Binomial theorem and equation 2, we obtain $\Delta \phi\left(n_{i}(g)+1\right)=p(1-p)\left[\delta a_{0}\left(p \delta a_{0}+1-p\right)^{n_{i}(g)}-\theta \delta a_{1}\left(p+\left(\delta a_{1}\right)(1-p)\right)^{n_{i}(g)}\right]$. We define $\gamma(\cdot, \cdot)$ as follows

$$
\gamma\left(n_{i}(g)+1, n_{j}(g)+1\right)=\Delta U_{i}(g, i j)=\Delta \phi\left(n_{i}(g)+1\right)-\Delta f_{1}\left(n_{i}(g)+1\right)-f_{2}\left(n_{j}(g)+1\right) .
$$


Clearly, $\gamma(\cdot, \cdot)$ is strictly decreasing in its first argument since $\Delta \phi(\cdot)$ is strictly decreasing by Proposition 2 and $\Delta f_{1}(\cdot)$ is strictly increasing. Similarly, $\gamma(\cdot, \cdot)$ is strictly decreasing in its second argument since $f_{2}(\cdot)$ is strictly increasing.

\subsection{Pairwise stable networks}

We first establish that there always exists a pairwise stable network in our setting (Proposition 3). Then we characterize these pairwise stable networks (Proposition 4).

To prove the existence result we use a theorem established by Erdös and Gallai, 1960. We need the following definition to present this theorem: A finite sequence $s=\left(d_{1}, d_{2}, \ldots, d_{n}\right)$ of nonnegative integers is graphical if there exists a network $g$ whose nodes have degrees $d_{1}, d_{2}, \ldots, d_{n}$.

Theorem 1 (Erdös and Gallai, 1960) A sequence $s=\left(d_{1}, d_{2}, \ldots, d_{n}\right)$ of nonnegative integers, such that $d_{1} \geq d_{2} \geq \ldots \geq d_{n}$, and whose sum is even is graphical if and only if

$$
\sum_{i=1}^{r} d_{i} \leq r(r-1)+\sum_{i=r+1}^{n} \min \left\{d_{i}, r\right\}, \text { for every } r, 1 \leq r<n .{ }^{12}
$$

In the following lemma we provide conditions that ensure the existence of three kinds of networks that turn out to be quite useful subsequently. Then, we show in Proposition 3 that one of these networks is always a pairwise stable network in our setting.

Lemma 1 Let $n$ and $k$ be nonnegative integers with $n>k$.

1. Let $n$ or $k$ be even. Then, the sequence $s=(k, \ldots, k)$ is graphical.

2. Let $n$ and $k$ be odds. Then, the sequences $s=(k+1, k \ldots, k), s^{\prime}=(k \ldots, k, k-1)$ are graphical.

Proof See Appendix

We now present our existence result. Let $k^{\star}, k^{\star} \in\{0, \ldots, n-1\}$, satisfy the two following conditions: (1) $\gamma\left(k^{\star}, k^{\star}\right) \geq 0$, and (2) there is no $k, k>k^{\star}$, such that $\gamma(k, k) \geq 0$. In other

\footnotetext{
${ }^{12}$ The theorem is also presented in Harary, 1969, Chapter 6 pp. 59-62 and the statement here is based on this presentation.
} 
words, $k^{\star}$ is the number of links which allows an agent $i$ who has formed $k^{\star}$ links to obtain a positive marginal expected payoff from a link with an agent $j$ who has formed $k^{\star}$ links. Furthermore, it is a threshold since no $k^{\prime}$ higher than $k^{\star}$ satisfies this property. Recall that $\gamma(\cdot, \cdot)$ is strictly decreasing in its two arguments. Hence, we have $\gamma(k, k)>0$ for all $k<k^{\star}$. Let $\eta(k)=\phi(k)-f_{1}(k)-k f_{2}(k)$. By assumption, $\phi(\cdot)$ is concave, and $f_{1}(\cdot)$ is convex. Moreover, $k f_{2}(k)$ is convex, since $f_{2}(\cdot)$ is increasing and convex. It follows that $\eta(\cdot)$ is concave and admits a unique maximum.

Proposition 3 Let the payoff function be given by equation 3. Then a pairwise stable network always exists.

Proof See Appendix.

Proposition 3 shows that our setting is consistent with our steady state solution of pairwise stability. The next proposition imposes conditions that a pairwise stable network must satisfy. In particular, this proposition does not exclude from the set of pairwise stable networks those networks where agents are in asymmetric positions relative to the amount of insurance they receive. To show this result, we need the following definition.

Let $S_{\ell}(g)=\left\{j \in N \mid n_{j}(g)=\ell\right\}$ be the set of agents who have formed $\ell$ links in $g$. It is worth noting that the set $S_{\ell}(g)$ is empty if no agents form $\ell$ links in $g .{ }^{13}$

Proposition 4 Let the payoff function be given by equation 3. If a network $g^{\star}$ is pairwise stable, then the two following conditions are satisfied:

1. (Q1) If $\ell, \ell^{\prime}>k^{\star}$, then there is no link between an agent who belongs to $S_{\ell}$ and an agent who belongs to $S_{\ell^{\prime}}$ in $g^{\star}$. If $\ell, \ell^{\prime}<k^{\star}$, then there is a link between an agent who belongs to $S_{\ell}$ and an agent who belongs to $S_{\ell^{\prime}}$ in $g^{\star}$.

2. (Q2) Suppose $\ell^{\prime} \leq \ell<k^{\star}$ and $k^{\star}<k^{\prime} \leq k$. If there is a link between an agent who belongs to $S_{\ell}$ and an agent who belongs to $S_{k}$ in $g^{\star}$, then there is a link between an agent who belongs to $S_{\ell^{\prime}}$ and an agent who belongs to $S_{k^{\prime}}$ in $g^{\star}$.

\footnotetext{
${ }^{13}$ We use $S_{\ell}(g)=S_{\ell}$ when there is no doubt about the network being studied.
} 
Proof Let $g^{\star}$ be a pairwise stable network. To introduce a contradiction, suppose that $g^{\star}$ does not satisfy property Q1. In particular, suppose that there is a link between an agent, say $i$, who belongs to $S_{\ell}$ and an agent, say $j$, who belongs to $S_{\ell^{\prime}}$, with $\ell, \ell^{\prime}>k^{\star}$, in $g^{\star}$. Then, the marginal payoff of agent $j$ from the link with agent $i$ is equal to $\gamma\left(n_{j}(g), n_{i}(g)\right)$ with $\left(n_{j}(g), n_{i}(g)\right) \geq\left(k^{\star}+1, k^{\star}+1\right)$. Since $\gamma$ is strictly decreasing in its two arguments, we have $\gamma\left(n_{j}(g), n_{i}(g)\right) \leq \gamma\left(k^{\star}+1, k^{\star}+1\right)<0$. It follows that the link between agent $i$ and $j$ does not belong to $g^{\star}$, a contradiction. Similarly, suppose that there is no link between an agent, say $i$, who belongs to $S_{\ell}$ and a agent, say $j$, who belongs to $S_{\ell^{\prime}}$, with $\ell, \ell^{\prime}<k^{\star}$, in $g^{\star}$. Then, the marginal payoff associated with the link between agents $i$ and $j$ for agent $j$ is equal to $\gamma\left(n_{j}(g), n_{i}(g)\right)$ with $\left(n_{j}(g), n_{i}(g)\right)<\left(k^{\star}, k^{\star}\right)$. Since $\gamma$ is strictly decreasing in its two arguments, we have $\gamma\left(n_{j}(g), n_{i}(g)\right)>\gamma\left(k^{\star}, k^{\star}\right) \geq 0$. Similarly, for agent $i$ we have $\gamma\left(n_{i}(g), n_{j}(g)\right)>\gamma\left(k^{\star}, k^{\star}\right) \geq 0$. It follows that agents $i$ and $j$ have an incentive to be linked in $g^{\star}$, a contradiction.

Next suppose that $g^{\star}$ does not satisfy property Q2. In particular, consider $\ell, \ell^{\prime}, k$, and $k^{\prime}$ such that $\ell^{\prime} \leq \ell<k^{\star}$ and $k^{\star}<k^{\prime} \leq k$ and suppose that there is a link between an agent, say $i$, who belongs to $S_{\ell}$ and an agent, say $j$, who belongs to $S_{k}$ in $g^{\star}$ and there is no link between an agent, say $i^{\prime}$, who belongs to $S_{\ell^{\prime}}$ and an agent, say $j^{\prime}$, who belongs to $S_{k^{\prime}}$ in $g^{\star}$. Since there is a link between agents $i$ and $j$ we have $\gamma\left(n_{i}(g), n_{j}(g)\right) \geq 0$ and $\gamma\left(n_{j}(g), n_{i}(g)\right) \geq 0$. Moreover, since there is no link between agents $i^{\prime}$ and $j^{\prime}$, we have $\gamma\left(n_{i^{\prime}}(g), n_{j^{\prime}}(g)\right)<0$ or $\gamma\left(n_{j^{\prime}}(g), n_{i^{\prime}}(g)\right)<0$ with $n_{i}(g) \geq n_{i^{\prime}}(g)$ and $n_{j}(g) \geq n_{j^{\prime}}(g)$. These inequalities are not compatible with the fact that $\gamma$ is strictly decreasing in its two arguments, a contradiction.

We now illustrate graphically Proposition 4. In Figure 2, network $g$ satisfies the properties given in $Q 1$. More precisely, in network $g, k^{\star}=4$, with $S_{5}=\{1,2\}, S_{4}=\{3,4,5\}$, and $S_{3}=\{6,7\}$. Moreover, agents who belong to $S_{5}, 5>k^{\star}$, are not linked together, and agents who belong to $S_{3}, 3<k^{\star}$, are linked together.

In Proposition 4 , we do not examine agents who have formed $k^{\star}$ links. Indeed, these agents can form links both with agents who have formed $x>k^{\star}$ links and with agents who have formed $y<k^{\star}$ links. 


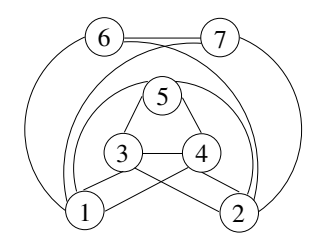

Figure 2: Network $g$

Proposition 4 highlights several properties of pairwise stable networks. Firstly, agents who obtain the highest amount of insurance are never linked together. This property illustrates the fact that some agents play a specific role in the provision of mutual insurance in the pairwise stable networks: these agents insure (and are insured by) a large part of the population. But an agent of this type does not interact with other agents of this type. In some sense there may exist "some insurance leaders" but these leaders themselves are not linked by mutual insurance agreements. Secondly, agents who obtain the smallest amount of insurance are always linked together. This property illustrates the fact that agents, who do not have a sufficient amount of insurance, will always reach mutual insurance agreements.

It follows from these two properties and the pigeon hole principle ${ }^{14}$, the following corollary.

Corollary 1 Suppose that the payoff function is given by equation 3. Then, agents who have formed a number of links higher than $k^{\star}$ (and obtain the highest amount of insurance) are always fewer than the rest of the population.

Lastly, pairwise stable networks may divide the population into sets of agents who are in asymmetric positions relative to their risk exposure. In other words, in a pairwise stable network, some agents are better off since they obtain insurance from others agents, who are involved in few mutual insurance agreements themselves. We now present an example which illustrates this property.

Example 1 Suppose $N=\{1, \ldots, 4\}, f_{1}\left(n_{i}(g)\right)=\phi\left(n_{i}(g)\right)-h\left(n_{i}(g)\right)$ with $h(0)=0, h(1)=4$,

\footnotetext{
${ }^{14}$ This principle is as follows. Let $n$ and $k$ be positive integers, and let $n>k$. Suppose we have to place $n$ identical balls into $k$ identical boxes, where $n>k$. Then there will be at least one box in which we place at least two balls.
} 
$h(2)=4.5, h(3)=4.75$ and the payoff function is given by equation 3. Moreover, $f_{2}(1)=0$, $f_{2}(2)=1, f_{2}(3)=3.5$. In this case, a star is pairwise stable.

Note that in our model, agents can have different numbers of bilateral insurance agreements, but this difference is bounded. Indeed, let $\bar{n}(g)=\max _{i \in N}\left\{n_{i}(g)\right\}$ be the number of links formed by the agents who have formed the highest number of links and let $\underline{n}(g)=\min _{i \in N}\left\{n_{i}(g)\right\}$ be the number of links formed by the agents who have formed the smallest number of links.

Corollary 2 Suppose that the payoff function is given by equation 3. Let $g^{\star}$ be a pairwise stable network. Then (a) $\bar{n}\left(g^{\star}\right) \leq \sum_{\ell \leq k^{\star}}\left|S_{\ell}\left(g^{\star}\right)\right|$ and (b) $\underline{n}\left(g^{\star}\right) \geq \sum_{\ell<k^{\star}}\left|S_{\ell}\left(g^{\star}\right)\right|$.

We now deal with networks where all agents obtain either an identical or a very similar amount of insurance. First, in the following corollary, we provide conditions which ensure that $k$-regular networks are pairwise stable networks.

Corollary 3 Suppose that the payoff function is given by equation 3. If $n$ or $k^{\star}$ are even, then there exists a k-regular network which is pairwise stable.

Proof This result follows the first part of the proof of Proposition 3.

Next we provide conditions which ensure that an almost- $k$-regular network is a pairwise stable network.

Corollary 4 Suppose that the payoff function is given by equation 3. Let $n$ and $k^{\star}$ be odd. Then, there exists an almost-k-regular network which is pairwise stable.

Proof This result follows the second part of the proof of Proposition 3.

Corollaries 3 and 4 establish that the set of pairwise stable networks always contains either a $k^{\star}$-regular network, or an almost- $k^{\star}$-regular network. The fact that agents are in "symmetric" positions in a pairwise stable mutual insurance network does not mean that these agents obtain the same amount of benefits. Indeed, in our model agents who draw 0 always obtain a lower amount of benefits than the benefits obtained by agents who draw 1 . 
Finally, we highlight the fact that in our setting agents can be partitioned into distinct components in a pairwise stable network.

Example 2 Suppose $N=\{1, \ldots, 12\}$ and $k^{\star}=2$. Then, network $g$ shown in Figure 3 is a pairwise stable network.

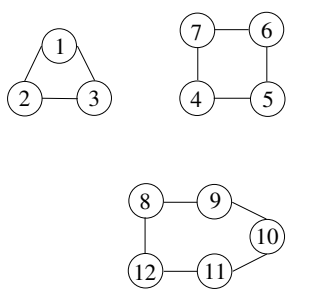

Figure 3: Network $g$

\subsection{Efficient Networks}

We now deal with the efficient networks. Note that the neighbors of agent $i$ are connected to an agent with $n_{i}(g)$ neighbors. Consequently, we have $W(g)=\sum_{i \in N}\left[\phi\left(n_{i}(g)\right)-f_{1}\left(n_{i}(g)\right)-\right.$ $\left.n_{i}(g) f_{2}\left(n_{i}(g)\right)\right]=\sum_{i \in N} \eta\left(n_{i}(g)\right)$. We know that $\eta(k)$ is concave. It follows that there exists $k^{e} \in\{0, \ldots, n-1\}$ such that $\eta(k)$ is maximal. Therefore $\sum_{i \in N} \eta\left(k^{e}\right) \geq \sum_{i \in N} \eta\left(n_{i}(g)\right)$ for all $n_{i}(g) \in\{0, \ldots, n-1\}$. Similarly, we have $\arg \max _{k \neq k^{e}} \eta(k) \subset\left\{k^{e}-1, k^{e}+1\right\}$ since $\eta(\cdot)$ is concave and maximum for $k=k^{e}$. Therefore, we have $\sum_{i=1}^{n-1} \eta\left(k^{e}\right)+\max \left\{\eta\left(k^{e}-1\right), \eta\left(k^{e}+\right.\right.$ $1)\} \geq \sum_{i=1}^{n-1} \eta\left(k^{e}\right)+\eta\left(n_{n}(g)\right)$ for $n_{n}(g) \neq k^{e}$. These observations are summarized in the next proposition.

Proposition 5 Suppose that the payoff function is given by equation 3. Let $g^{e}$ be an efficient network. Then, $g^{e}$ is either a $k^{e}$-regular network, or an almost-k $k^{-}$-regular network.

Proof We use Lemma 1 to ensure the existence of one of these three networks.

It follows from Corollary 3 that if $k$ or $n$ are even, then only $k^{\star}$-regular networks can be both pairwise stable and efficient. However, in the following corollary we show that $k^{\star}$-regular 
stable networks and efficient networks do not always coincide. More precisely, we establish that in the stable network, that is the $k^{\star}$-regular network, agents will form at least the same number of links as in the efficient network. In this case, a non-efficient pairwise stable network is always over-connected from the efficiency perspective. To simplify the presentation, we assume that $n$ is even.

Corollary 5 Suppose that the payoff function is given by equation 3. Suppose $n$ is even. Then in the $k^{\star}$-regular stable network agents will form at least the same number of links as in the efficient network.

Proof Suppose $n$ is even. Then by Lemma 1, network $g^{\star}$ where all agents have formed $k^{\star}$ links, and network $g^{e}$ where all agents have formed $k^{e}$ links, exist. By Corollary 3, we know that $g^{\star}$ is pairwise stable, and by Proposition 5, we know that $g^{e}$ is efficient. Moreover, we have $\gamma(k, k)-(\eta(k)-\eta(k-1))=(k-1)\left(f_{2}(k)-f_{2}(k-1)\right) \geq 0$, for $0<k<n$ since $f_{2}$ is strictly increasing. Now, by definition of $k^{e}$, we have $\eta\left(k^{e}\right)-\eta\left(k^{e}-1\right) \geq 0$. It follows that we have $\gamma\left(k^{e}, k^{e}\right) \geq \eta\left(k^{e}\right)-\eta\left(k^{e}-1\right) \geq 0$. We know that by definition of $k^{\star}$, we have $\gamma(k, k)<0$, for all $k>k^{\star}$. It follows that $k^{\star}$ is at least equal to $k^{e}$.

The intuition behind this result is as follows. Let $g^{\star}$ be the regular stable network where all agents have formed $k^{\star}$ links, and let $i, j, k$ be three agents such that $g_{i j}^{\star}=g_{i k}^{\star}=1$, and $g_{k j}^{\star}=0$. If agents $i$ deletes her link with agent $j$, then agent $k$ will benefit from this deletion, since her payoff will increase by $f_{2}\left(n_{i}\left(g^{\star}\right)-1\right)-f_{2}\left(n_{i}\left(g^{\star}\right)\right)$. However, when $i$ decides whether to delete her link with $j$ in $g^{\star}$, she does not take into account the positive externality that would accrue to $k$ from the deletion of this link.

\section{Pairwise stable networks with generous and miserly agents}

Till now, we have assumed that all agents who draw 1 give the same amount of resources, $\delta$, to their neighbors who draw 0 . We now consider an extension of our benchmark model where this assumption is relaxed. In particular, one can imagine that agents do not have the same 
preferences with respect to the generosity of the transfer scheme. Some agents could give large amount of resources to their unlucky neighbors while some others only give them small amount of resources.

We will assume that the population can be partitioned into two sets: the set of generous agents, $N^{G}$, and the set of miserly agents, $N^{M} \cdot{ }^{15}$ If a generous agent draws state 1 , then she gives $\delta^{G}$ to each of her neighbors who draws state 0 . Similarly, if a miserly agent draws state 1 , then she gives $\delta^{M}$ to each of her neighbors who draws state 0 . Obviously, we assume that $\delta^{G}>\delta^{M}$.

Since this framework is harder to analyze than the previous one, we restrict attention to the linear cost function; we assume that agent $i \in N$ incurs a cost $F$ for each link she forms. ${ }^{16}$ Note that with miserly and generous agents, our analysis faces the following problem: the ENB function given by equation 2 is not well-defined since $b^{g}(0, k)$ will now depend on the order of the terms in which $\delta^{G}$ and $\delta^{M}$ appear in the sum.

To define an expected neighborhood benefits function consistent with this new framework, we have to present some additional definitions. For each $m \in\{1, \ldots, n-1\}$, we define $X^{m}(\alpha)$ as the set of vectors which belong to $\left\{\delta^{M}, \delta^{G}\right\}^{m}$ such that $\alpha$ of their elements are equal to $\delta^{G}$ and $m-\alpha$ are equal to $\delta^{M}$. We assume that each order of $\delta^{M}$ and $\delta^{G}$ has the same probability of being drawn. Since there are $m ! /(\alpha !(m-\alpha) !)$ distinct vectors in $X^{m}(\alpha)$, each of them has a probability $(\alpha !(m-\alpha) !) / m$ ! of occurrence. For each $X^{m}(\alpha)$ we define the following function $\varphi_{\alpha}^{m}:\left(x_{0}, \ldots, x_{m}\right) \in X^{m}(\alpha) \mapsto \varphi_{\alpha}^{m}\left(x_{0}, \ldots, x_{m}\right)=\sum_{j=0}^{m}\left(x_{j} a_{0}\right)^{j}$, with $x_{j} a_{0} \in(0,1)$.

We now assume that each agent has the same probability of drawing a 0 or 1: it does not depend on her preference regarding the transfer scheme. Moreover, if there are $\alpha \geq 0$ agents who belong to $N^{G}$ in the neighborhood of $i$ and $k$ agents in $N_{i}(g)$ who draw 1 , then there is a probability given by

$$
\mathcal{Q}(\alpha, x, k)=\left[\left(\begin{array}{l}
\alpha \\
x
\end{array}\right)\left(\begin{array}{c}
n_{i}(g)-\alpha \\
k-x
\end{array}\right)\right] /\left[\sum_{\ell=0}^{k}\left(\begin{array}{l}
\alpha \\
\ell
\end{array}\right)\left(\begin{array}{c}
n_{i}(g)-\alpha \\
k-\ell
\end{array}\right)\right]
$$

\footnotetext{
${ }^{15}$ We have $N=N^{G} \cup N^{M}, N^{M} \cap N^{G}=\emptyset$ and $N^{M}, N^{G} \neq \emptyset$.

${ }^{16}$ It is possible to do the analysis with the cost function assumed in section 2, but the analysis would be harder and would not provide any additional insights.
} 
such that $x, x \leq \alpha$, of these $k$ agents belong to $N^{G}$.

We now define $\bar{b}^{g}(0, \alpha, k)$, which describes the expected benefits obtained by $i$ in $g$ when she draws $0, \alpha$ agents in her neighborhood are generous, and $k$ agents in her neighborhood draw 1, as follows:

$$
\bar{b}^{g}(0, \alpha, k)=\sum_{y=0}^{k} \mathcal{Q}(\alpha, y, k)\left[\sum_{\ell \in X^{k}(y)}\left(\frac{y !(k-y) !}{k !}\right) \varphi_{y}^{k}(\ell)\right] \cdot{ }^{17}
$$

It is worth noting that if there exists only one type of agents, then both definitions of $b^{g}(0, \cdot)$ and $\bar{b}^{g}(0, \cdot, \cdot)$ are "equivalent". Indeed, given that the size of the neighborhood of agent $i$ is $n_{i}(g)$, if we assume that all agents in the neighborhood of agent $i$ are generous and give $\delta=\delta^{G}$ to their neighbors, then we have $\bar{b}^{g}\left(0, n_{i}(g), k\right)=b^{g}(0, k)$. Similarly, if we assume that all agents in the neighborhood of agent $i$ are miserly and give $\delta=\delta^{M}$ to their neighbors, then $\bar{b}^{g}(0,0, k)=b^{g}(0, k)$.

We now define the expected neighborhood benefits function of agent $i \in N^{x}, x \in\{G, M\}$, given that her neighborhood contains $n_{i}(g)$ agents and $\alpha$ among them are generous agents.

$$
\begin{aligned}
\bar{B}_{i}(g)=\bar{\phi}\left(n_{i}(g), \alpha\right)= & p \sum_{k=0}^{n_{i}(g)}\left(\begin{array}{c}
n_{i}(g) \\
k
\end{array}\right) p^{k}(1-p)^{n_{i}(g)-k} b_{x}^{g}(1, k) \\
& +(1-p) \sum_{k=0}^{n_{i}(g)}\left(\begin{array}{c}
n_{i}(g) \\
k
\end{array}\right) p^{k}(1-p)^{n_{i}(g)-k} \bar{b}^{g}(0, \alpha, k),
\end{aligned}
$$

where $b_{x}^{g}(1, k)$ is equal to $b^{g}(1, k)$ when agent $i$ gives $\delta=\delta^{x}, x \in\{G, M\}$, to each of her neighbors who draw 0 . In the following, we are interested in the incentives of agent $i \in N^{x}$ to form a link with an agent $j \in N^{y}, y \in\{G, M\}$, given that the cost associated with each link is $F$.

We denote by $\mathcal{Z}_{i, j}^{x, y}(g)=\bar{B}_{i}\left(g+g_{i, j}\right)-\bar{B}_{i}(g)$ the marginal expected neighborhood benefits obtained by agent $i \in N^{x}, x \in\{G, M\}$, when she forms a link with an agent $j \in N^{y}$, $y \in\{G, M\}$, in $g$.

Proposition 6 Suppose that the expected neighborhood benefits is given by equation 6 and the cost of each link is $F$. Then, $\mathcal{Z}_{i, j}^{G, G}(g)>\mathcal{Z}_{i, j}^{G, M}(g)$, and $\mathcal{Z}_{i, j}^{M, G}(g)>\mathcal{Z}_{i, j}^{M, M}(g)$.

\footnotetext{
${ }^{17}$ This function takes into account all orders that can appear, and each order has the same weight as the others. Qualitatively, it is very close to the idea used in the probabilistic version of the Shapley value.
} 
Proof We only prove that $\mathcal{Z}_{i, j}^{G, G}(g)>\mathcal{Z}_{i, j}^{G, M}(g)$, since $\mathcal{Z}_{i, j}^{M, G}(g)>\mathcal{Z}_{i, j}^{M, M}(g)$ is established using the same arguments. Let $g^{G}$ be the network which is identical to $g$ except that agent $i \in N^{G}$ has formed an additional link with agent $j \in N^{G}$, and let $g^{M}$ be the network which is identical to $g$ except that agent $i \in N^{G}$ has formed an additional link with agent $j^{\prime} \in N^{M}$. Let $\alpha^{G}=\left|N_{i}\left(g^{G}\right) \cap N^{G}\right|$ and $\alpha^{M}=\left|N_{i}\left(g^{M}\right) \cap N^{G}\right|$. We have $\alpha^{G}=\alpha^{M}+1$. Moreover, we have $\mathcal{Z}_{i, j}^{G, G}(g)-\mathcal{Z}_{i, j^{\prime}}^{G, M}(g)=(1-p) \sum_{k=0}^{n_{i}(g)}\left(\begin{array}{c}n_{i}(g) \\ k\end{array}\right) p^{k}(1-p)^{n_{i}(g)-k}\left(\bar{b}^{g^{G}}\left(0, \alpha^{G}, k\right)-\bar{b}^{g^{M}}\left(0, \alpha^{M}, k\right)\right)$. Suppose that $k$ agents respectively in $N_{i}\left(g^{G}\right)$ and in $N_{i}\left(g^{M}\right)$ draw 1 . Then, we define $H(x)=$ $\left.\mathcal{Q}\left(\alpha^{G}, x, k\right) / \mathcal{Q}\left(\alpha^{M}, x, k\right)=\left[\left(\begin{array}{c}\alpha_{G} \\ x\end{array}\right)\left(\begin{array}{c}n_{i}(g)+1-\alpha_{G} \\ k-x\end{array}\right)\right] /\left[\begin{array}{c}\alpha_{M} \\ x\end{array}\right)\left(\begin{array}{c}n_{i}(g)+1-\alpha_{M} \\ k-x\end{array}\right)\right]$ which provides the ratio of the weights (when they are defined) associated with the different vectors which belong to $\left\{\delta^{M}, \delta^{G}\right\}^{n_{i}(g)+1} . H(x)$ is an increasing function with the number of agents who draw state 1 in the neighborhood of agent $i$. Consequently, the weights associated with the most valuable vectors in $X^{n_{i}(g)+1}\left(\alpha^{G}\right)$ are higher than the weights associated with the most valuable vectors in $X^{n_{i}(g)+1}\left(\alpha^{M}\right)$. It follows that $\mathcal{Z}_{i, j}^{G, G}(g)>\mathcal{Z}_{i, j^{\prime}}^{G, M}(g)$.

To simplify the analysis, we now assume that $\left|N^{G}\right|=\left|N^{M}\right|$. In the following proposition, we establish that there exist parameters such that a network where agents are linked only with other agents of the same type is a pairwise stable network. We denote by $g^{G / M}$ the network such that $g_{i, j}^{G / M}=1$ if and only if $i, j \in N^{x}, x \in\{G, M\}$. The network $g^{G / M}$ can be seen as two different networks: the complete network $g_{1}^{G /}$ which contains all agents in $N^{G}$ and where $g_{i, j}^{G /}=g_{i, j}^{G / M}$ for all $i, j \in N^{G}$, and the complete network $g^{/ M}$ which contains all agents in $N^{M}$ and where $g_{i, j}^{/ M}=g_{i, j}^{G / M}$. The ENB of each player is not the same in $g^{G /}$ and in $g^{/ M}$ since agents do not give and receive the same amount of money in each of these networks. Let $\Delta \bar{\phi}^{G /}=$ $\bar{\phi}\left(\left|N^{G}\right|-1,\left|N^{G}\right|-1\right)-\bar{\phi}\left(\left|N^{G}\right|-2,\left|N^{G}\right|-2\right)$ be the marginal ENB obtained by agent $i \in N^{G}$ in network $g^{G /}$. Similarly, we denote by $\Delta \bar{\phi}^{/ M}=\bar{\phi}\left(\left|N^{M}\right|-1,\left|N^{M}\right|-1\right)-\bar{\phi}\left(\left|N^{M}\right|-2,\left|N^{M}\right|-2\right)$ the marginal ENB obtained by agent $i \in N^{M}$ in network $g^{/ M}$.

Proposition 7 Suppose that the expected neighborhood benefits is given by equation 6 and the cost of each link is $F$. Then, there exists $F>0$ such that $g^{G / M}$ is a pairwise stable network.

Proof We assume that $\min \left\{\Delta \bar{\phi}^{G /}, \Delta \bar{\phi}^{/ M}\right\}>F>\mathcal{Z}_{i, j}^{G, M}\left(g^{G / M}\right)$. We show that $g^{G / M}$ is pairwise stable. The possibility of $\Delta \bar{\phi}^{/ M}>\mathcal{Z}_{i, j}^{G, M}\left(g^{G / M}\right)$ is guaranteed by the concavity of 
$\bar{\phi}^{G /}(\cdot), \bar{\phi}^{/ M}(\cdot)$ and the fact that we can choose any value for $\left|N^{G}\right|$ and $\left|N^{M}\right|$.

First, since $F>\mathcal{Z}_{i, j}^{G / M}\left(g^{G / M}\right)$, no agent in $N^{G}$ will accept to form a link with an agent in $N^{M}$. Consequently, there is no agent $i \in N^{G}$ who has an incentive to form an additional link in $g^{G / M}$.

Second, since $\Delta \bar{\phi}^{G /}>F$ and $\Delta \bar{\phi}^{/ M}>F$, there does not exist any agent $i \in N$ who has an incentive to remove one of her links in $g^{G / M}$.

We now illustrate through an example that there exist situations where the only pairwise stable network is a network where generous agents form links only with all other generous agents, while miserly agents form links only with all other miserly agents. This equilibrium result is compatible with a result stressed by several empirical studies. Indeed, Rosenzweig, 1988, and Udry, 1994, find that the majority of transfers takes place only between sub-groups of agents.

Example 3 We assume that $N=\{1, \ldots, 4\}$ with $\{1,2\} \in N^{G}$ and $\{3,4\} \in N^{M}$. Moreover, we assume that $\delta^{G}=0.1, \delta^{M}=0.08, a_{0}=2, a_{1}=13, \Theta=1, \theta=1 / 1000$, and $p=3 / 4$. The payoff of agents 1 and 2 are identical when they form the same links. Moreover, the link $g_{1,3}$ plays the same role as the link $g_{1,4}$ for the ENB of agent 1 . In Table 1 we establish the ENB obtained by agent 1 in interesting networks with regard to the characterization of pairwise stable networks. Similarly, the payoff of agents 3 and 4 are identical when they form the same links. Moreover, the link $g_{3,1}$ plays the same role as the link $g_{3,2}$ for the ENB of agent 3 . In Table 2 we establish the ENB obtained by agent 3 in interesting networks with regard to the characterization of pairwise stable networks. 


\begin{tabular}{|l|r|r|l|}
\hline$N_{1}(g)$ & Draw 1 & Draw 0 & ENB \\
\hline$\emptyset$ & 1 & 0 & 0.75 \\
\hline$\{2\}$ & 0.99968 & 0.15 & 0.78726 \\
\hline$\{3\}$ & 0.99968 & 0.12 & 0.77976 \\
\hline$\{3,4\}$ & 0.99933 & 0.1644 & 0.79059 \\
\hline$\{2,3\}$ & 0.99933 & 0.1872 & 0.79629 \\
\hline$\{2,3,4\}$ & 0.99895 & 0.19855 & 0.79885 \\
\hline
\end{tabular}

Table 1. ENB obtained by agent 1.

\begin{tabular}{|l|r|r|l|}
\hline$N_{3}(g)$ & Draw 1 & Draw 0 & ENB \\
\hline$\emptyset$ & 1 & 0 & 0.75 \\
\hline$\{1\}$ & 0.99974 & 0.15 & 0.78731 \\
\hline$\{4\}$ & 0.99974 & 0.12 & 0.77981 \\
\hline$\{1,2\}$ & 0.99948 & 0.21 & 0.80211 \\
\hline$\{1,4\}$ & 0.99948 & 0.1872 & 0.79641 \\
\hline$\{1,2,4\}$ & 0.99921 & 0.21628 & 0.80348 \\
\hline
\end{tabular}

Table 2. ENB obtained by agent 3 .

In the first column of Table 1 (Table 2), we indicate the agents with whom agent 1 (agent 3 ) has formed a link. In the second column of Table 1 (Table 2) we indicate the ENB that agent 1 (agent 3) obtains if she draws state 1 . In the third column of Table 1 (Table 2) we indicate the ENB that agent 1 (agent 3) obtains if she draws state 0 . The fourth column of Table 1 (Table 2) indicates the ENB obtained by agent 1 (agent 3). Clearly, if $F \in(0.02977,0.02979)$, then the network, which contains only a link between agent 1 and 2 , and a link between agent 3 and 4 , is the unique pairwise stable network.

Let us now explain why it is possible to obtain the network, where generous agents have links only with other generous agents while miserly agents have links only with other miserly agents, as the only pairwise stable network. Clearly, each agent $i$ prefers to form a link with agents who belong to $N^{G}$ to form a link with agents who belong to $N^{M}$. Moreover, there exists a cost of forming links $F$ such that an agent $i \in N^{M}$ accepts to form a link with an agent $j \in N^{M}$ while an agent $i^{\prime} \in N^{G}$ does not accept such a link. Indeed, agents $i \in N^{M}$ and $i^{\prime} \in N^{G}$ obtain the same marginal expected profit from a link with an agent $j \in N^{M}$, when they draws state 0. But agent $i \in N^{M}$ incurs a smaller marginal expected loss from a link with $j \in N^{M}$ than agent $i^{\prime} \in N^{G}$, when they draws state 1 . Moreover, by Proposition 2 and the fact that both definitions of $b^{g}(0, \cdot)$ and $\bar{b}^{g}(0, \cdot, \cdot)$ are "equivalent" if there exists only one type of agents, the ENB is concave in a network where agents are partitioned according to their type. Since $\mathcal{Z}_{i, j}^{G, G}(g)>\mathcal{Z}_{i, j}^{G, M}(g)$, this concavity property is preserved when an agent $i^{\prime} \in N^{G}$ has formed links only with other agents in $N^{G}$ and forms a first link with an agent $i \in N^{M}$. Consequently, it is sufficient to find conditions such that an agent $i^{\prime} \in N^{G}$ has an incentive to form a link 
with an agent $j^{\prime} \in N^{G}, i^{\prime} \in N^{G}$ has no incentive to form a link with $i \in N^{M}$, and an agent $i \in N^{M}$ has an incentive to form a link with $j \in N^{M}$ to obtain the required example.

It is worth noting that for sufficiently small costs of setting links generous agents consent to form links with miserly agents. Indeed, generous agents are risk averse and prefer to be linked with miserly agents to be less insured.

\section{Conclusion}

In this paper, we examine the option that agents can make some mutual informal insurance arrangements on their own. More precisely, agents come to agreements with their neighbors concerning a transfer scheme: each agent helps her neighbors who draw state 0 when she draws state 1 and each agent is helped by her neighbors who draw state 1 when she herself draws state 0 .

We find that efficient networks are either $k$-regular networks, or almost-k-regular networks. In other words, only networks where agents obtained a very similar level of insurance are efficient networks. By contrast, there exist conditions such that asymmetric networks, in terms of the insurance they provide to the agents, are pairwise stable.

Finally, we extend our model to situations where there exist two kinds of agents: generous ones and miserly ones. We highlight that there exist parameters under which generous agents have links only with other generous agents while miserly agents have links only with other miserly agents in a pairwise stable network.

\section{Appendix}

To simplify notation, we extend $b^{g}(\cdot, \cdot)$ to $b^{g}(1,-1)=\Theta-\theta \sum_{j=0}^{n_{i}(g)+1}\left(\delta a_{1}\right)^{j}$. To demonstrate Propositions 1 and 2, we need to compute the difference between $B_{i}\left(g+g_{i j}\right)$ and $B_{i}(g)$, called $\Delta B_{i}\left(g, g_{i j}\right)$. We set $P\left(n_{i}(g), k\right)=\left(\begin{array}{c}n_{i}(g) \\ k\end{array}\right) p^{k}(1-p)^{n_{i}(g)-k}$.

Sketch of Proof of Proposition 1. We present successively the two situations which can 
arise when agent $i$ forms an additional link with agent $j$ in $g$.

Suppose $j$ draws 1 . This occurs with probability $p$. Then the benefits obtained by agent $i$ when she forms a link with agent $j$ is

$$
\left(p \sum_{k=0}^{n_{i}(g)} P\left(n_{i}(g), k\right) b^{g}(1, k)\right)+\left((1-p) \sum_{k=0}^{n_{i}(g)} P\left(n_{i}(g), k\right) b^{g}(0, k+1)\right)
$$

Suppose $j$ draws 0 . This occurs with probability $1-p$. Then the benefits obtained by agent $i$ when she forms a link with $j$ is

$$
\left(p \sum_{k=0}^{n_{i}(g)} P\left(n_{i}(g), k\right) b^{g}(1, k-1)\right)+\left((1-p) \sum_{k=0}^{n_{i}(g)} P\left(n_{i}(g), k\right) b^{g}(0, k)\right)
$$

Straightforward computations show that

$$
\begin{aligned}
\Delta B_{i}\left(g, g_{i j}\right) & =p(1-p)\left\{\sum_{k=0}^{n_{i}(g)} P\left(n_{i}(g), k\right)\left(\delta a_{0}\right)^{k+1}-\sum_{k=0}^{n_{i}(g)} P\left(n_{i}(g), k\right) \theta\left(\delta a_{1}\right)^{n_{i}(g)-k+1}\right\} \\
& =p(1-p)\left\{\sum_{k=0}^{n_{i}(g)}\left(\begin{array}{c}
n_{i}(g) \\
k
\end{array}\right) p^{k}(1-p)^{n_{i}(g)-k}\left[\left(\delta a_{0}\right)^{k+1}-\theta\left(\delta a_{1}\right)^{n_{i}(g)-k+1}\right]\right\} .
\end{aligned}
$$

Since $\left(\delta a_{1}\right)^{n}<(1 / \theta)\left(\delta a_{0}\right)^{n}, \delta a_{0}<1$ and $\delta a_{1}>1$, we have $\left(\delta a_{0}\right)^{k+1} \geq\left(\delta a_{0}\right)^{n}>\theta\left(\delta a_{1}\right)^{n} \geq$ $\theta\left(\delta a_{1}\right)^{n_{i}(g)-k+1}$, for all $n_{i}(g) \in\{0, \ldots, n-1\}$, and $k \in\left\{0, \ldots, n_{i}(g)-1\right\}$. Therefore, $B_{i}(g+$ $\left.g_{i j}\right)-B_{i}(g)>0$.

Sketch of Proof of Proposition 2. In order to prove this statement we need to assign a sign to the difference between the marginal benefits. To obtain this result, we assume that agent $i$ adds the link $g_{i k}=1$ to the network $g+g_{i j}$. Following the same steps as in Proposition 1 we have for $\Delta B_{i}\left(g+g_{i j}, g_{i k}\right)=B_{i}\left(g+g_{i j}+g_{i k}\right)-B_{i}\left(g+g_{i j}\right)$ :

$$
\begin{aligned}
\Delta B_{i}\left(g+g_{i j}, g_{i k}\right)= & p(1-p)\left(\sum_{k=0}^{n_{i}(g)+1} P\left(n_{i}(g)+1, k\right)\left(\delta a_{0}\right)^{k+1}\right. \\
& \left.-\sum_{k=0}^{n_{i}(g)+1} P\left(n_{i}(g)+1, k\right) \theta\left(\delta a_{1}\right)^{n_{i}(g)-k+2}\right) .
\end{aligned}
$$

We now determine the sign of the difference between $\Delta B_{i}\left(g+g_{i j}+g_{i k}\right)$ and $\Delta B_{i}\left(g+g_{i j}\right)$. Following the same steps as in Proposition 1, we obtain that the sign of $\Delta B_{i}\left(g+g_{i j}+g_{i k}\right)-$ $\Delta B_{i}\left(g+g_{i j}\right)$ is equal to the sign of:

$$
\delta a_{0}\left(p \delta a_{0}+1-p\right)^{n_{i}(g)}\left(p\left(\delta a_{0}-1\right)\right)-\theta \delta a_{1}\left(\delta a_{1}-1\right)(1-p)\left(p+\left(\delta a_{1}\right)(1-p)\right)^{n_{i}(g)} .
$$


Since $\delta a_{0}<0$ and $\delta a_{1}>1$ we have $\Delta B_{i}\left(g+g_{i j}, g_{i k}\right)-\Delta B_{i}\left(g, g_{i j}\right)<0$.

Proof of Lemma 1. We prove successively that the three sequences are graphical.

1. Suppose $n$ or $k$ is even. Let $n>k>0$. Since either $n$, or $k$ is even, the sum of the sequence $s=(k, k \ldots, k)$ is even. Equation 5 can be written as

$$
r k \leq r(r-1)+\sum_{i=r+1}^{n} \min \{k, r\}, \text { for every } r, 1 \leq r<n .
$$

There are two cases. Suppose $r \leq k$. Then equation 8 is satisfied if

$$
r k \leq r(r-1)+(n-r) r \Rightarrow k \leq(r-1)+(n-r) \Rightarrow k \leq n-1 .
$$

This equation is always satisfied. Suppose $r>k$. Then equation 8 is

$$
r k \leq r(r-1)+(n-r) k
$$

If $k=n-1$, then $s=(n-1, \ldots, n-1)$ is a graphical sequence since the complete network supports this sequence. Similarly, if $k=0$, then $s=(0, \ldots, 0)$ is a graphical sequence since the empty network supports this sequence. We now deal with $k, 0<k<n-1$. We have

$$
r(r-1)+(n-r) k-r k=r^{2}-r(1+2 k)+n k=\left(r-\frac{2 k+1}{2}\right)^{2}+n k-\left(\frac{2 k+1}{2}\right)^{2} .
$$

Since $n k \geq(k+2) k=(k+1) k+k$ and $\left(\frac{2 k+1}{2}\right)^{2}=(k+1) k+1 / 4$, we have $n k-\left(\frac{2 k+1}{2}\right)^{2} \geq 0$, for $0<k<n-1$.

2. Suppose $n$ and $k$ are odd, with $n-1>k>0(k \neq n-1$ since $k$ and $n$ are odd $)$. Since $n$ is odd, $n-1$ is even and since $k$ is odd, $k+1$ is even. Consequently, the sum of the sequence $s=(k+1, k \ldots, k)$ is even.

For $r=1$, equation 5 is satisfied since $k+1 \leq(n-1)$ for $0<k<n-1$. For $r \geq 2$, equation 5 is equal to

$$
k+1+(r-1) k \leq r(r-1)+\sum_{i=r+1}^{n} \min \{k, r\}, \text { for every } r, 2 \leq r<n .
$$


There are two cases. (1) Suppose $r \leq k$, with $k \leq n-2$, and $r \geq 2$. Then equation 10 is $k+1+(r-1) k \leq r(r-1)+(n-r) r \Rightarrow k \leq(r-1)+(n-r)-\frac{1}{r} \Rightarrow k \leq(n-1)-\frac{1}{r}$.

This equation is always satisfied since $k<n-1$ and $1 / r<1$ for $r>2$. (2) Suppose $r>k$. Then equation 10 is

$$
r k+1 \leq r(r-1)+(n-r) k
$$

We first deal with the case where $k=n-2$. In that case $r=n-1$. Therefore, we have:

$$
(n-1)(n-2)+1 \leq(n-1)(n-2)+n-2,
$$

and since $n \geq 3$, this equation is always satisfied. We now deal with $k<n-2$, we have $r(r-1)+(n-r) k-r k-1=r^{2}-r(1+2 k)+n k-1=\left(r-\frac{2 k+1}{2}\right)^{2}+n k-\left(\frac{2 k+1}{2}\right)^{2}-1$.

Since $n k \geq(k+3) k=(k+1) k+2 k$ and $\left(\frac{2 k+1}{2}\right)^{2}+1=(k+1) k+5 / 4$, we have $n k-\left(\frac{2 k+1}{2}\right)^{2}-1>0$, for $0<k<n-2$.

3. Suppose $n$ and $k$ are odd. Since $n$ is odd, $n-1$ is even and since $k$ is odd, $k-1$ is even. Consequently, the sum of the sequence $s=(k, \ldots, k, k-1)$ is even. equation 5 is equal to

$$
r k \leq r(r-1)+\sum_{i=r+1}^{n-1} \min \{k, r\}+\min \{k-1, r\}, \text { for every } r, 1 \leq r<n-1 .
$$

There are two cases. (1) Suppose $r \leq k-1$, with $k<n-1(k \neq n-1$ since $n$ and $k$ are odd). Then equation 12 becomes

$$
r k \leq r(r-1)+(n-r) r, \text { for every } r, 1 \leq r<n .
$$

We have already shown in point 1 ., equation 9 , that this equation is always satisfied. (2) Suppose $r>k-1$. Then equation 12 becomes

$$
r k \leq r(r-1)+(n-r) k-1 \Rightarrow r k+1 \leq r(r-1)+(n-r) k .
$$


We first deal with the case where $k=n-2$. In that case either $r=n-1$, or $r=n-2$. We have shown in point 2. , equation 11 , that the previous equation is satisfied when $r=n-1$ and $k=n-2$. If $r=n-2$ and $k=n-2$, we have $(n-2)(n-2)+1 \leq(n-2)(n-3)+2(n-2) \Rightarrow(n-2)(n-2)+1 \leq(n-2)(n-2)+(n-2)$.

This equation is always satisfied since $n \geq 3$. Finally, we have shown in point 2., equation 11, that equation 13 is satisfied when $0<k<n-2$.

Proof of Proposition 3. Let $n$ or $k^{\star}$ be even. Then, we build the network $g^{r}$ where all agents form $k^{\star}$ links. We know by Lemma 1 that $g^{r}$ exists. We now show that $g^{r}$ is pairwise stable. First, no agent has a strict incentive to remove a link since $\gamma\left(k^{\star}, k^{\star}\right) \geq 0$ in $g^{r}$. Second, no agent has an incentive to add a link since $\gamma\left(k^{\star}+1, k^{\star}+1\right)<0$ in $g^{r}$. Therefore $g^{r}$ is a pairwise stable network.

Suppose now that $k$ and $n$ are odd. There are two cases: either (a) $\gamma\left(k^{\star}+1, k^{\star}\right) \geq 0$ and $\gamma\left(k^{\star}, k^{\star}+1\right) \geq 0$, or (b) $\gamma\left(k^{\star}+1, k^{\star}\right)<0$ or $\gamma\left(k^{\star}, k^{\star}+1\right)<0$. We first deal with case (a): $\gamma\left(k^{\star}+1, k^{\star}\right) \geq 0$ and $\gamma\left(k^{\star}, k^{\star}+1\right) \geq 0$, with $k^{\star} \neq 0$. We build the network $g$ where one agent, say $i$, forms $k^{\star}+1$ links and all other agents form $k^{\star}$ links. By Lemma 1 , the network $g$ exists. Using the same argument as above we can see that no agent has an incentive to add or remove one link in $g$. Next, we deal with case (b): $\gamma\left(k^{\star}+1, k^{\star}\right)<0$ or $\gamma\left(k^{\star}, k^{\star}+1\right)<0$, with $k^{\star} \neq 0$. We build the network $g^{\prime}$ where one agent, say $i$, forms $k^{\star}-1$ links and all other agents form $k^{\star}$ links. By Lemma 1, the network $g^{\prime}$ exists. If agent $i$ forms a link with agent $j$, then $i$ obtains a marginal payoff associated with this link equal to $\gamma\left(k^{\star}, k^{\star}+1\right)$, while $j$ obtains a marginal payoff associated with this link equal to $\gamma\left(k^{\star}+1, k^{\star}\right)$. By assumption, $\min \left\{\gamma\left(k^{\star}, k^{\star}+1\right), \gamma\left(k^{\star}+1, k^{\star}\right)\right\}<0$. Therefore agent $i$ or agent $j$ has no incentive to form this link. Moreover, no agent has an incentive to remove one of her links and no agent $j \in N \backslash\{i\}$ has an incentive to add a link with an agent $j^{\prime} \in N \backslash\{i\}$ in $g^{\prime}$, since $\gamma\left(k^{\star}+1, k^{\star}+1\right)<0$. This completes the proof. 